\title{
Prix, contrats et bourses de recherche décernés par la Société française de médecine d'Urgence (SFMU) en 2017
}

\author{
Awards, Scholarship and Research Grants Allocated by the French Society of Emergency \\ Medicine (SFMU) in 2017
}

\author{
S. Beaune $\cdot$ J. Truchot $\cdot$ M. Maignan $\cdot$ Pour la Commission recherche de la SFMU \\ Reçu le 21 décembre 2017; accepté le 21 décembre 2017 \\ (C) SFMU et Lavoisier SAS 2018
}

\section{Contrats recherche}

La SFMU en partenariat avec l'industrie invite chaque année les médecins et les paramédicaux exerçant en structure d'urgence à concrétiser un projet d'étude par l'attribution de contrats de recherche. Ces contrats ont pour objectif de promouvoir des projets de recherche clinique ou expérimentale par un soutien financier d'un montant de 5000 à $10000 €$.

\author{
S. Beaune $(\square)$ \\ Service d'accueil des urgences, \\ hôpitaux universitaires \\ Paris Île-de-France-Ouest, Ambroise Paré, \\ assistance publique hôpitaux de Paris (APHP), \\ F-92100 Boulogne-Billancourt, France \\ e-mail : sebastien.beaune@aphp.fr \\ Université Paris-Diderot, UMR-S INSERM 1144, \\ F-75006 Paris, France \\ J. Truchot \\ Service d'accueil des urgences, SMUR, \\ hôpital Lariboisière, APHP, \\ 2, rue Ambroise-Paré, F-75010 Paris, France \\ Université Paris-Diderot, Paris \\ M. Maignan \\ Service d'accueil des urgences, SAMU-SMUR, \\ centre hospitalier universitaire Grenoble-Alpes, \\ F-38043 Grenoble, France \\ Université Grenoble Alpes, UMR INSERM 1042, Grenoble \\ Pour la Commission recherche de la SFMU \\ Société française de médecine d'urgence (SFMU)*, \\ 103, boulevard Magenta, F-75010 Paris, France
}

\footnotetext{
* La Société française de médecine d'urgence (SFMU) propose chaque année un soutien à la recherche clinique sous forme de bourses et de contrats recherche ainsi que des prix récompensant les meilleurs travaux.
}

\section{Bourse de recherche non thématique}

L'objectif de cette bourse est le soutien d'un candidat investi en médecine d'urgence et ayant le projet d'une activité de recherche dans une structure opératoire, de pilotage ou de gestion de recherche clinique ou fondamentale dans le domaine de la médecine d'urgence. Cette activité de recherche peut avoir lieu en France ou à l'étranger comme la réalisation d'une année de recherche «mobilité », dans le cadre d'un Master 2 ou d'une thèse d'université. Elle est dotée d'un montant de $20000 €$ grâce au soutien du fonds de recherche de la SFMU et de ses partenaires de l'industrie.

Dans les deux cas, le jury est constitué des membres de la commission recherche et d'au moins un membre du conseil d'administration de la SFMU. Comme chaque année, la commission scientifique et la commission soins et urgences de la SFMU ont attribué des prix, les « U », aux meilleurs résumés proposés au congrès Urgences 2017.

En 2017, un contrat et deux bourses de recherche ont été attribués ainsi que plusieurs prix :

- un contrat d'un montant de $9300 €$ a été attribué au Dr Julien Fonsegrive (service d'accueil des urgences du $\mathrm{CH}$ Le Mans) pour son projet concernant l'intérêt d'un anesthésique topique à base de lidocaïne et de prilocaïne (crème ATLP) dans la prise en charge des plaies superficielles aux urgences, «Étude Douleur \& Plaies ». L'objectif principal de cette étude est d'évaluer l'efficacité, sur la douleur maximale ressentie pendant les soins, de l'application de crème ATLP sur la plaie dès l'accueil par rapport à l'injection sous-cutanée de lidocaïne adrénalinée avant la suture. Les objectifs secondaires sont l'évaluation de l'efficacité de l'application de crème ATLP sur les plaies superficielles dès l'accueil par rapport à l'injection sous-cutanée de lidocaïne adrénalinée avant suture en termes de : satisfaction du patient, taux d'échec de l'anesthésie, douleur aux différents moments de la prise en charge, temps de prise en charge. 
Il s'agit d'une étude prospective, multicentrique, randomisée, ouverte et contrôlée visant à inclure 126 patients sur la période d'étude ;

- un contrat d'un montant de $6450 €$ a été attribué au Dr Quentin Le Bastard (service d'accueil des urgences du CHU de Nantes) pour son projet concernant l'impact de l'antibiothérapie des pyélonéphrites communautaires sur le portage digestif de bactéries multirésistantes. Il s'agit d'une étude expérimentale et interventionnelle sur un modèle murin. L'objectif principal de cette étude est d'évaluer le taux de colonisation digestive par une entérobactérie résistante après l'exposition à une stratégie antibiotique de désescalade (prescription successive de deux antibiothérapies) ou à une stratégie de monothérapie antibiotique sans traitement relais. Les objectifs secondaires sont l'identification longitudinale des modifications de la flore digestive en termes de diversité et de genres bactériens résistants après l'exposition à une stratégie antibiotique de désescalade (prescription successive de deux antibiothérapies) ou à une stratégie de monothérapie antibiotique sans traitement relais, l'évaluation du taux de colonisation digestive par une entérobactérie résistante après l'exposition aux différentes stratégies d'antibiothérapie de relais, l'identification des genres bactériens associés à l'acquisition d'une souche bactérienne résistante. Il s'agit d'une étude expérimentale avec six groupes d'animaux ;

- la bourse non thématique d'un montant de $20000 €$ a été décernée au Dr Charles-Henri Houzé-Cerfon (pôle de médecine d'urgence, CHU de Toulouse) pour un projet portant sur la simulation en santé pour accompagner le changement pédagogique par l'évaluation de dispositifs d'apprentissage, des professionnels de santé, aux situations critiques. Cette étude en sciences de l'éducation et médecine vise la comparaison de l'effet de deux modalités de débriefing post-simulation sur l'apprentissage des compétences non techniques mobilisées dans la gestion d'une situation critique en santé en équipe interprofessionnelle : débriefing combiné (associant un débriefing individuel et un débriefing collectif) et débriefing collectif conventionnel. La durée totale de cette étude sera de 18 mois et 60 professionnels seront inclus par quatre centres.
Concernant les prix, le «U d'or médecin » a été décerné aux Drs Yonathan Freund, Alexandra Rousseau, Adeline Aubry, François-Pierre Auffredou, Pierre-Alexis Raynal, Anne-Laure Feral-Pierssens, Tabassome Simon, Bruno Riou (Paris, Bobigny) pour leur étude intitulée " Règle PERC pour exclure le diagnostic d'embolie pulmonaire aux urgences : l'essai randomisé contrôlé PROPER »; le «U d'or soignant » a été attribué à Judith Leblanc, Gaëlle Duchene, Cécile Lancien, Christine Jauneau, Théophile Bastide, Rachel Verbrugghe, Hélène Piquet, Anne-Claude Cremieux (Paris, Saclay, Bobigny, Gonesse, St Denis, Garches) pour l'étude "Le dépistage infirmier ciblé du VIH dans huit services d'urgence».

Le «U d'argent médecin » a été attribué aux Drs. Hélène Goulet, Alexandra Rousseau, Maxime Maignan, Sabine Guinemer, Patrick Ray, Tabassome Simon, Bruno Riou, Yonathan Freund (Paris, Grenoble, Bobigny) pour l'étude «Vérifications croisées systématiques aux urgences pour diminuer le taux d'erreurs médicales: l'étude randomisée contrôlée CHARMED »; le «U d'argent soignant » a été attribué à Célia Lococo, Sabine Bressand, Adrien Zakine, Mehdi Khellaf, Lionel Nakad, Virginie Ferrando, Anthony Tuytten (Créteil) pour l'étude « Évaluation de la pertinence d'une voie veineuse périphérique et l'intérêt d'un prolongateur à valve bidirectionnelle ».

Le « $\mathrm{U}$ de bronze médecin » a récompensé les Drs JeanMarc Agostinucci, Carla De Stefano, Catherine Pradeaux, Géraldine Gonzalez, Philippe Bertrand, Aurélié Avondo, Louis Soulat, Frédéric Lapostolle (Bobigny, Bordeaux, Dijon, Châteauroux) pour l'étude «Perception de l'arrêt cardiaque par les collégiens. Facteurs liés à l'intention de porter secours en situation d'urgence : une étude exploratoire transversale » et le « $\mathrm{U}$ de bronze soignant » a été attribué à Marie-Laure Poujade, Jean-Arnaud Elissalde, Anouck Burg-Martinez, Nathalie Urcullu, Florian Despeysses, Laurent Maillard (Agen) pour l'étude « Connaissances théoriques et expertise des infirmiers des urgences face à la prise en charge des personnes âgées de 75 ans et plus dans un service d'accueil et d'urgence en France : étude pilote».

Les prochaines récompenses seront remises au cours de l'assemblée générale de la SFMU lors du congrès Urgences 2018. 